\title{
Secure Online Transaction using Iris
}

\author{
K. Sivasankari, Abhishek Balamurugan, Sai Dhanush. R, Sundeep. J.
}

\begin{abstract}
In this project, we are planning to create a strong robust calculation for executing cash in higher level security reason with high acknowledgment rates in a shifting environment. To begin with, Haar cascade based calculation has been connected for quick and basic confront location from the input picture. The confront picture is at that point being changed over into grayscale picture. After that, the iris, eyebrows, nose, mouth of candidates are extricated from the escalated valleys from the recognized confront.
\end{abstract}

Keywords: Adaboost learning, Biometric Verification Software, Haar Cascade, Secure Electronic Transaction.

\section{INTRODUCTION}

$I^{2}$ the last few days, we see a dramatic increase in the use of advanced technology in different areas to change and interact with our customers. This is often very true when talking about the banking sector. From the starting of the digital evolution identity verification has been gaining prominent role over the time because of the convenience it offers and the main reason for that is not compromising on the safety of transactions. Even though there is an increase in using of digital transaction like using cards, e-wallets and net banking etc., combined with the creation of the safe and strong password there are still many cases of fraudulent transaction occurring in the banking sector. Even after many changes in policies of the banks still we are losing many billions from reputed banking institutes due to this fraud, so to keep a check to this fraud cases there has been an increase in usage of biometric and facial recognition rather than using the password policies which is easy to hack. Now all the banking institutes are creating software which rely on the biometric and facial scans of the customer and then storing it in the personal system and then using it for verification process for the transaction. The main motto of this is to verify the customer's identity and to allow the transaction complete only if the customer's verification matches the bank's records.

Manuscript received on 29 September 2021 | Revised Manuscript received on 05 October 2021 | Manuscript Accepted on 15 November 2021 | Manuscript published on 30 November 2021.

* Correspondence Author

Abhishek Balamurugan*, B. Tech, Department of Computer Science from SRM University Chennai, (Tamil Nadu) India.

Sai Dhanush. R, B. Tech, Department of Computer Science from SRM University Chennai born and raised in Chennai, (Tamil Nadu) India.

Sundeep. J, B. Tech, Department of Computer Science from SRM University Chennai born and raised in Chennai, (Tamil Nadu) India.

Sivasankari. K, Assistant Professor, Department of Computer Science Engineering, SRMIST, Ramapuram Campus, Chennai, (Tamil Nadu) India.

(c) The Authors. Published by Lattice Science Publication (LSP). This is an open access article under the CC-BY-NC-ND license (http://creativecommons.org/licenses/by-nc-nd/4.0/)

\section{A. Problem Definition}

The biometric verification software with additional banking software could be more conventional methods. In fact, using passwords is not the only solution for the issue. And also customers will be creating the password which is very easy to remember. It is a piece of cake for the cyber attackers to use few tricks to get what they wanted. Adding to that peoples will be having many passwords to remember for e.g. for Facebook, Instagram, and other online application which requires password. And, when making difficult passwords, people forget their passwords. There is only one way to change a forgotten password. there is only one way that is sending the password changing code to the registered mobile number or registered mail id which is easy to hacker, they can intercept the inbox and use the same ode for their purpose. But if the bank institute implements this biometric and facial recognition method the hacker can't have the access to the facial recognition because the customers have only one face which allow them access the transaction.

\section{B. Project Description}

Facial recognition is one in all varied ways in which banks will decrease friction in their customers' expertise and increase potency and accessibility. This project creates identification and Account Withdrawals permitting customers to form withdrawals from their bank accounts. Bio facial recognition packages can minimize fraud if online hackers illegally uses customers password and follows one another way to steal at banking facilities. The program package verifies the identity of the individual before processing the transaction. Our goal is to produce associate particularly resistance, customized expertise attentively on security

\section{Objective}

The facial authentication program system contains active spotting to put a stop to the online hackers on using the spoofing function of the client's image. It also has relevance to alternative biometrics. This is only if survival detection specifically does it. Assess the 'survival' of the registered face image as a result of the stored data. In addition, the Authentication program allows bank customers to verify their accounts details of the bank from the comfort of their home by using internet connectivity. Biometric verification program is one of the many ways banks can reduce the friction of their customers' expertise and increase their effectiveness and accessibility.

\section{SYSTEM ANALYSIS}

\section{A. Existing System}

In previous days they used only single level authentication like OTP generation. it had been less secured.

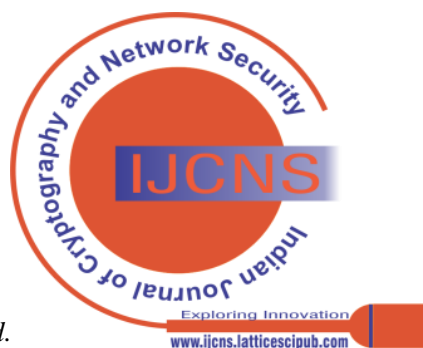




\section{Secure Online Transaction using Iris}

Secure Electronic Transaction (SET) includes various levels of encryption using symmetric encryption, asymmetric encryption and many combinations of hashes. Since not all agents have their own secret key, the only problem remaining is public key distribution, but cardholders can determine asymmetric keys.

\section{B. Disadvantages}

The bank customers should and must have a MasterCard, and when the payment is low, no profit will be made. There is no anonymity, which can be traced back to trading users who must install a separate client software. The cost and complexity of support provided by merchants is in sharp contrast to the relatively low cost and ease of use of current SSL-based alternatives. Client certificate distribution logistics.

\section{Proposed System}

This uses machine learning techniques to induce a high degree of accuracy from what's called "training data". Haar Cascades use the Adaboost learning algorithm which selects a tiny low number of important features from an oversized set to offer an efficient results of classifiers. Initially, the algorithm requires many positive pictures (face images) and negative pictures (no face images) to train the program classifiers. Therefore, we have to extract features from it. To do this, use the Haar characteristic shown below the image. they're a bit like our convolutional kernel. Each feature may be a single value obtained by subtracting sum of pixels under white rectangle from sum of pixels under black rectangle.

\section{Advantage}

A. The main reason why Haar cascade algorithm is used majorly is because of its calculation speed.

B. Haar Cascade is a type of object/ particle detection algorithm which majorly is used in image or video in which the object is detected and is further analysed.

C. Haar Cascades uses an Adaboost learning method that selects a small number of important characteristics in a large set to produce efficient sorter output. (a) Edge Features
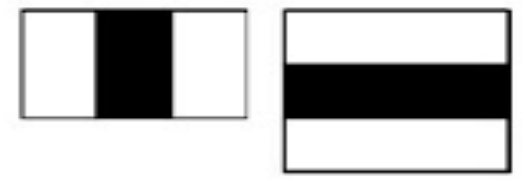

(b) Line Features

(c) Four-rectangle features

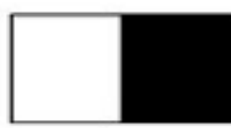

\author{
Features
}

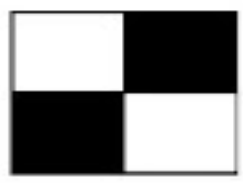




\section{E. System Design}

\section{A. SYSTEM ARCITECTURE}

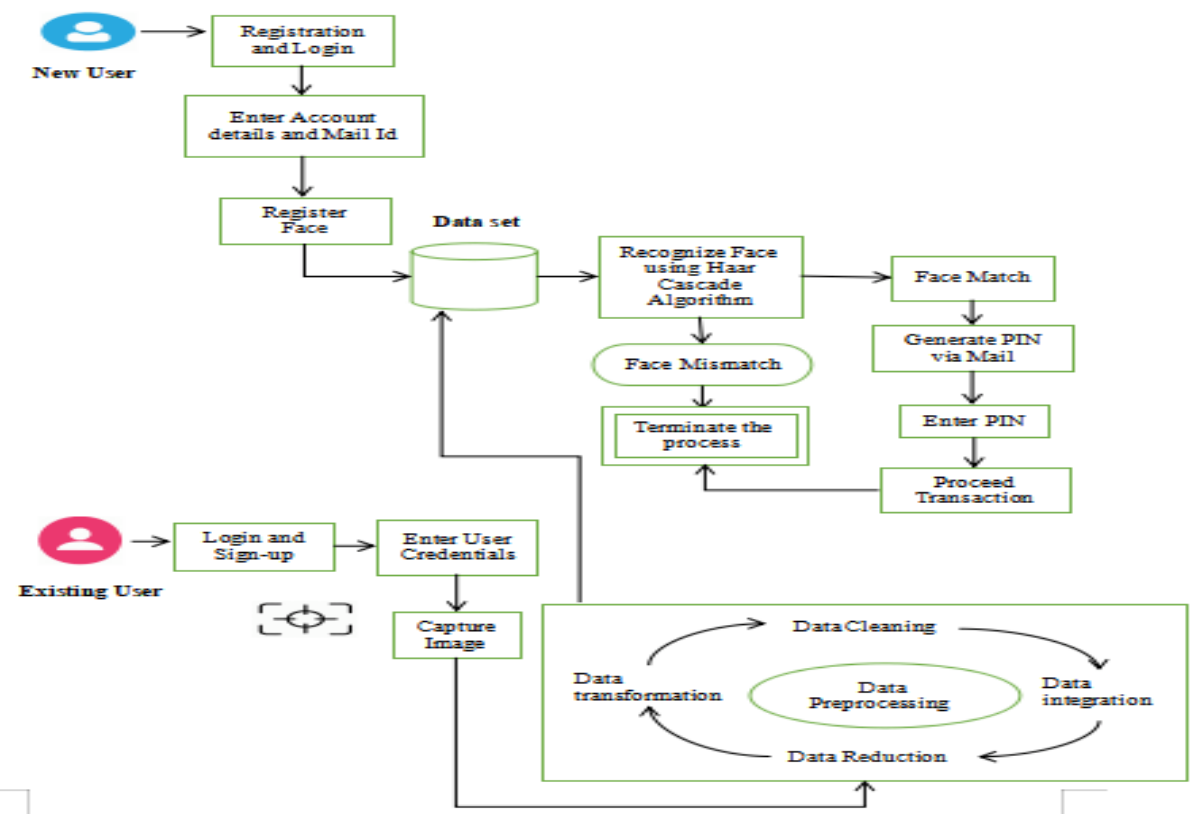

\section{F. DATA FLOW DIAGRAM}

A. Bubble chart is another name for DFD. Received data is a basic graphic format which can be used to depict the system on the basis of the processing performed on that data and the data generated in the output.

B. One of the most important modeling tools is the data flow diagram (DFD). It is used to represent the many components of the system. System Processes External entities that interact with data systems used in processes and the flow of information from the system are examples of all these components.

DFD-0

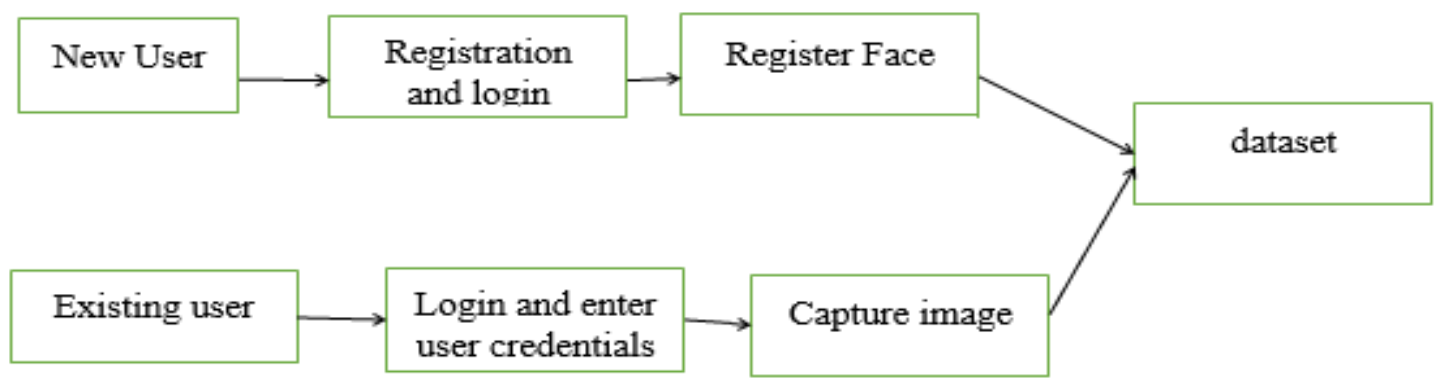

DFD-1

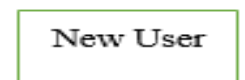

Registration and login

Existing user
Login and enter user credentials

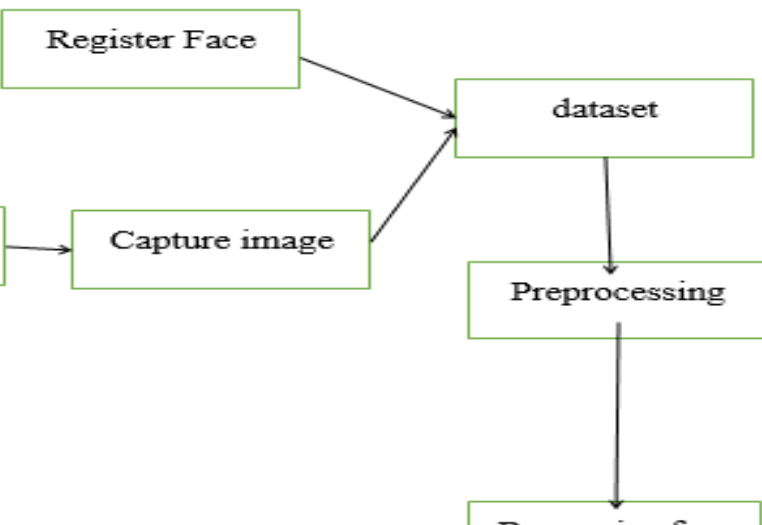

Recognize face

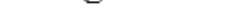$$
\text { . }
$$

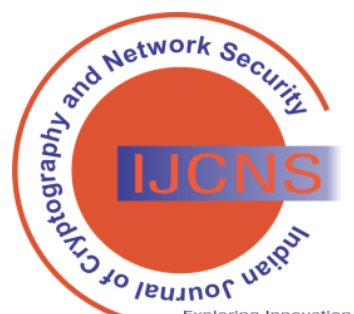

Published By: 


\section{Secure Online Transaction using Iris}

DFD2

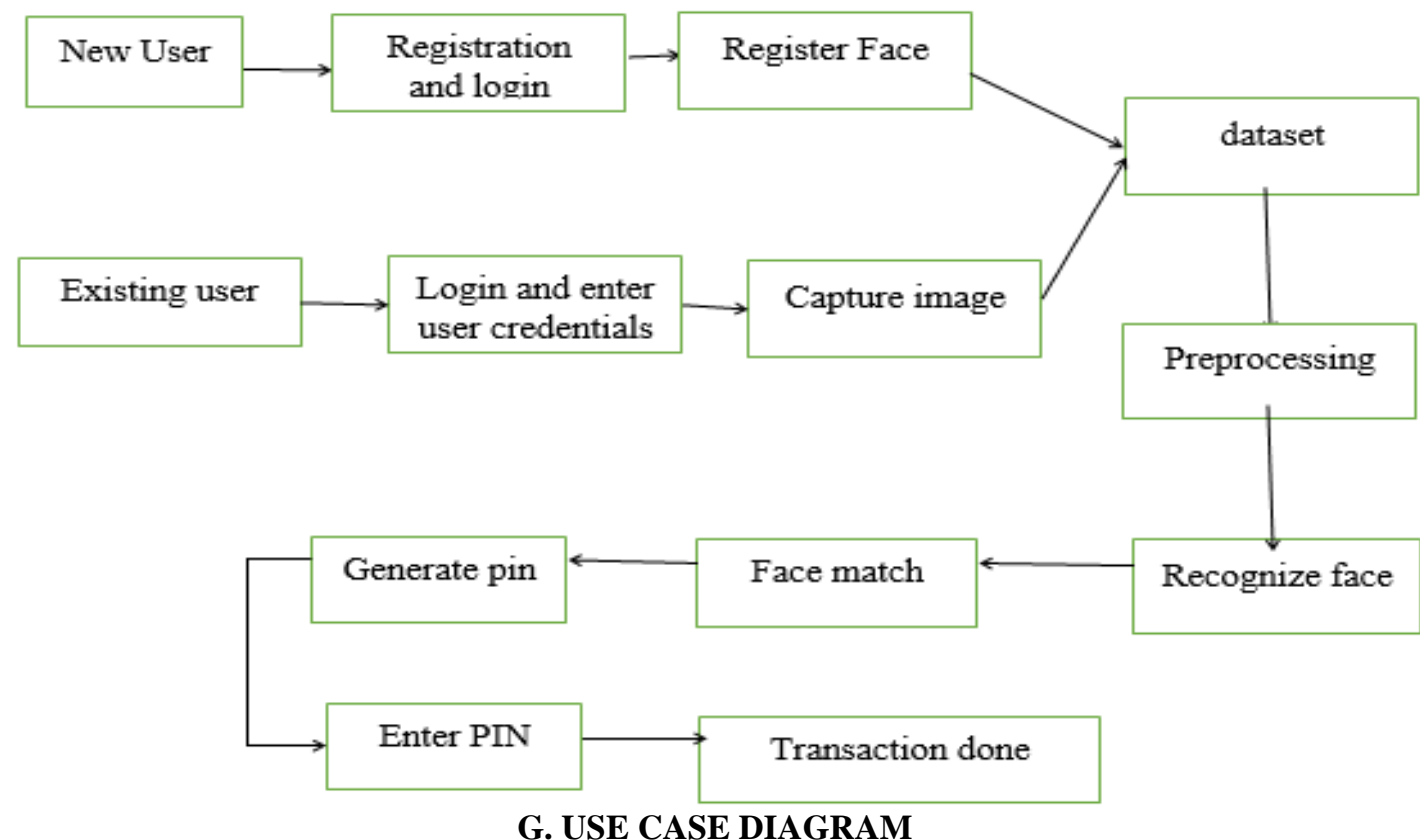

It is a specified form of behavior diagram and it is derived from the analysis in the Unified Modeling Language (UML). Its objective is to provide a graphical representation of the functionalities of the system in terms of actors, objectives (represented in the form of use cases) and any dependencies between the use cases. The main purpose of a use case diagram is to show which actors the functions of the system are performed to. The roles of the actors of the system can be displayed.

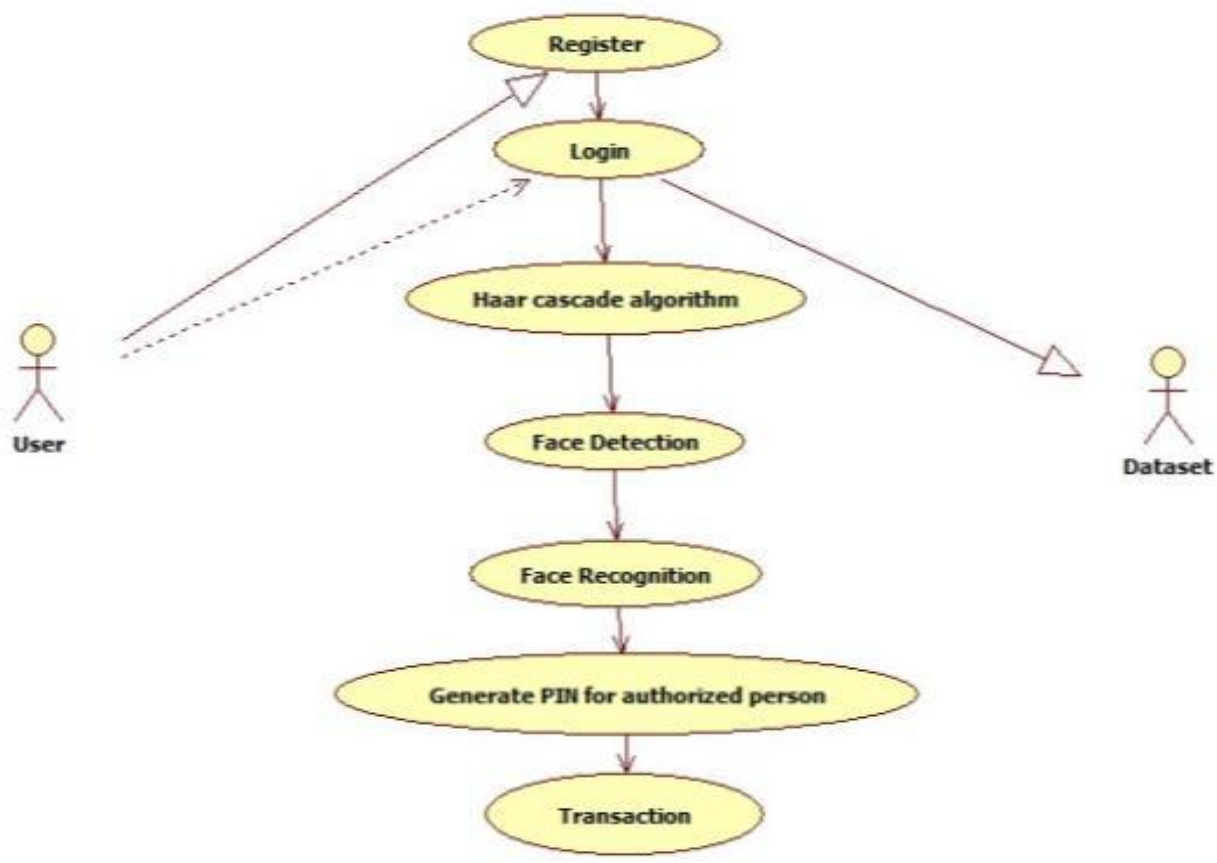

\section{H. SEQUENCE DIAGRAM}

UML (Unified Modeling Language) sequence diagrams are collaborative diagrams that illustrate how processes interact amongst each other. The data flow diagram's structure. Event diagrams, event scenarios, and sequence diagrams are all names for sequence diagrams.

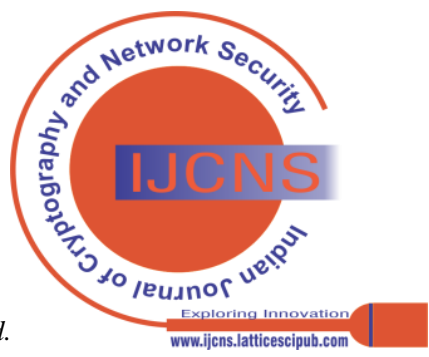




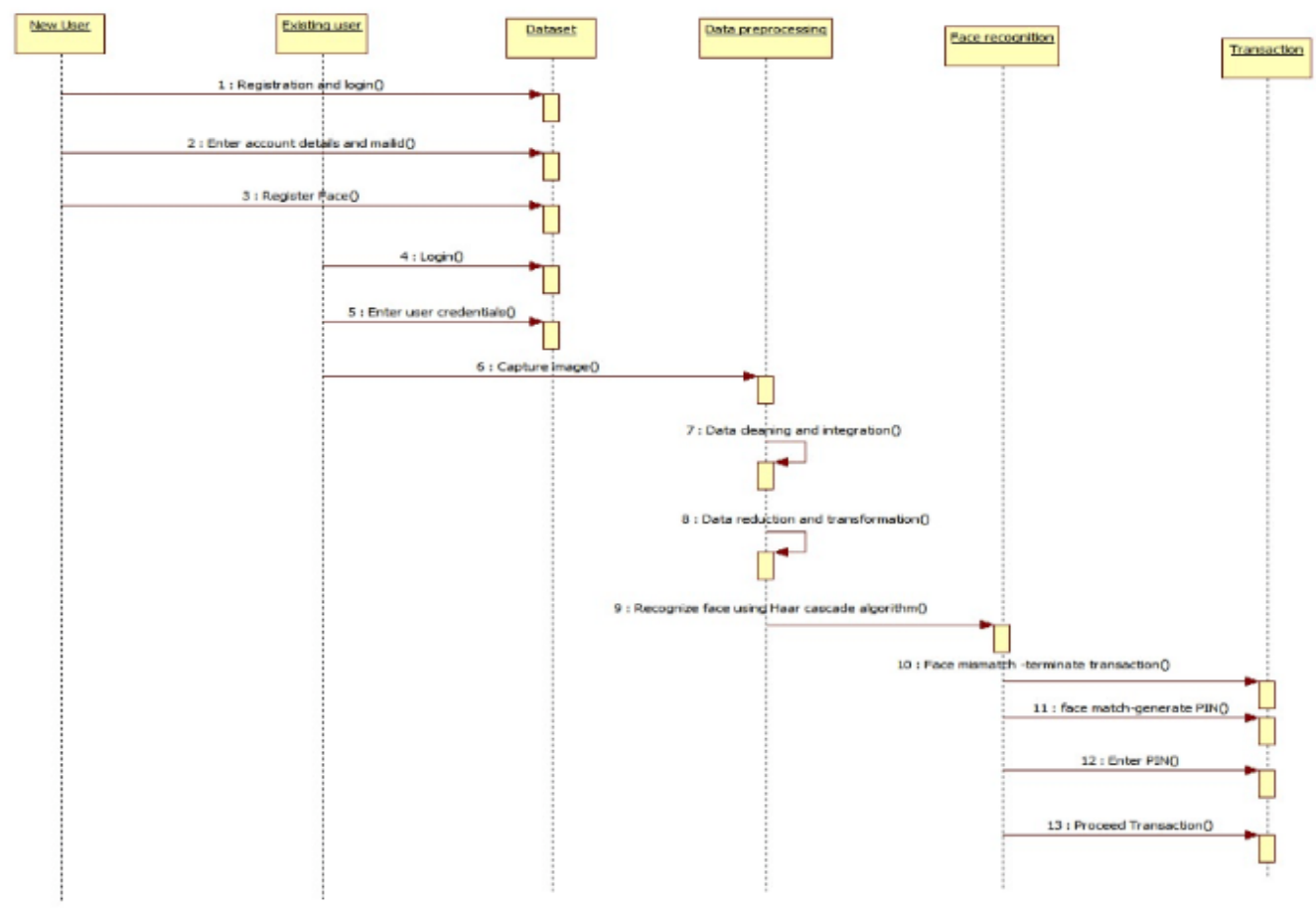

\section{ACTIVITY DIAGRAM:}

An activity diagram is a visual depiction of a process that allows for the selection, repetition, and synchronization of activities and tasks in a process approach. In an integrated modelling language, you may use activity diagrams to describe the strategic and technical process focus of sustainable components. The whole data stream is represented in the activity diagram.
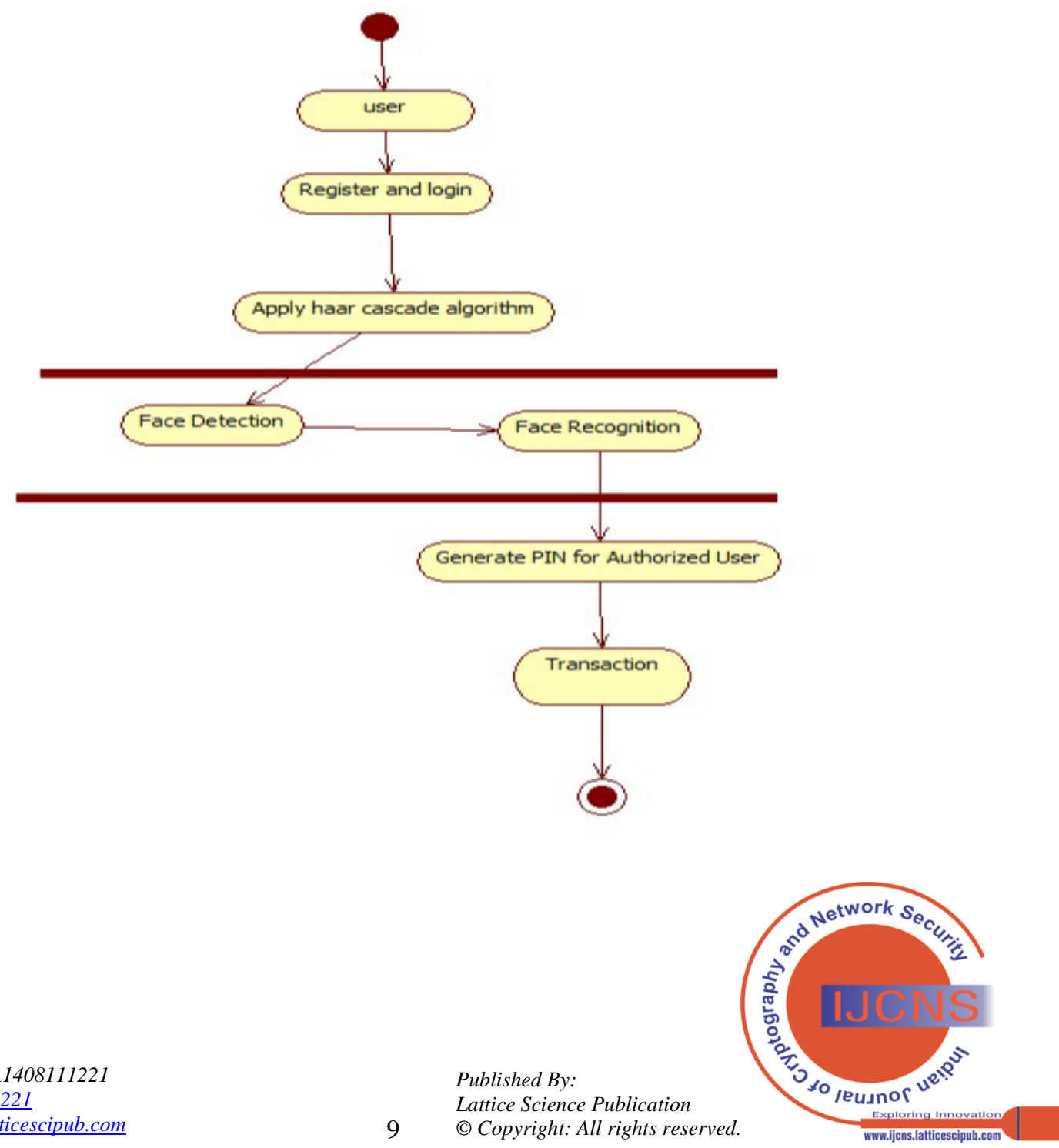


\section{Secure Online Transaction using Iris}

\section{J. CLASS DIAGRAM}

The class diagram's goal is to offer a basic perspective of the system. Class diagrams seem to be the only illustrations which can be connected directly in an object-oriented programming, making them particularly popular in the building process. Class diagrams are dynamic diagrams. This object is represented a pictorial structure of the program. Class diagrams can be used to also depict, describe, and record many elements of a system, but also to develop source codes for application software.

\section{IMPLEMENTATION}

\section{A. Modules}

1. Data Preprocessing

2. Feature Extraction

3. Face Recognition

B. Data Pre-processing

A. This is a method for converting ambiguous data into clear data sources.

B. That is, when information is gathered from several resources, it is imported in its original format, making analysis difficult.

\section{Feature extraction}

It's the process of converting an image's raw pixel values into more relevant information that may be used in other approaches like point matching or machine learning.

\section{Face Recognition}

Authentication process, business transactions, and Kiosks are all excellent uses for facial authentication program. Face code authentication program is capable of doing the following tasks:

a. Locate dynamically changing in the area of vision of the camera.

b. checks to see if the movement entity is in front of the camera.

c. Angle compares the active faces to database samples.

\section{E. Register}

Registration module user enter their details for registration into the system.

\section{F. Transaction}

In this phase the transaction is proceed when the face is matched with the registered user otherwise the transaction is terminated.

\section{IMPLEMENTATION SCREENSHOT:}
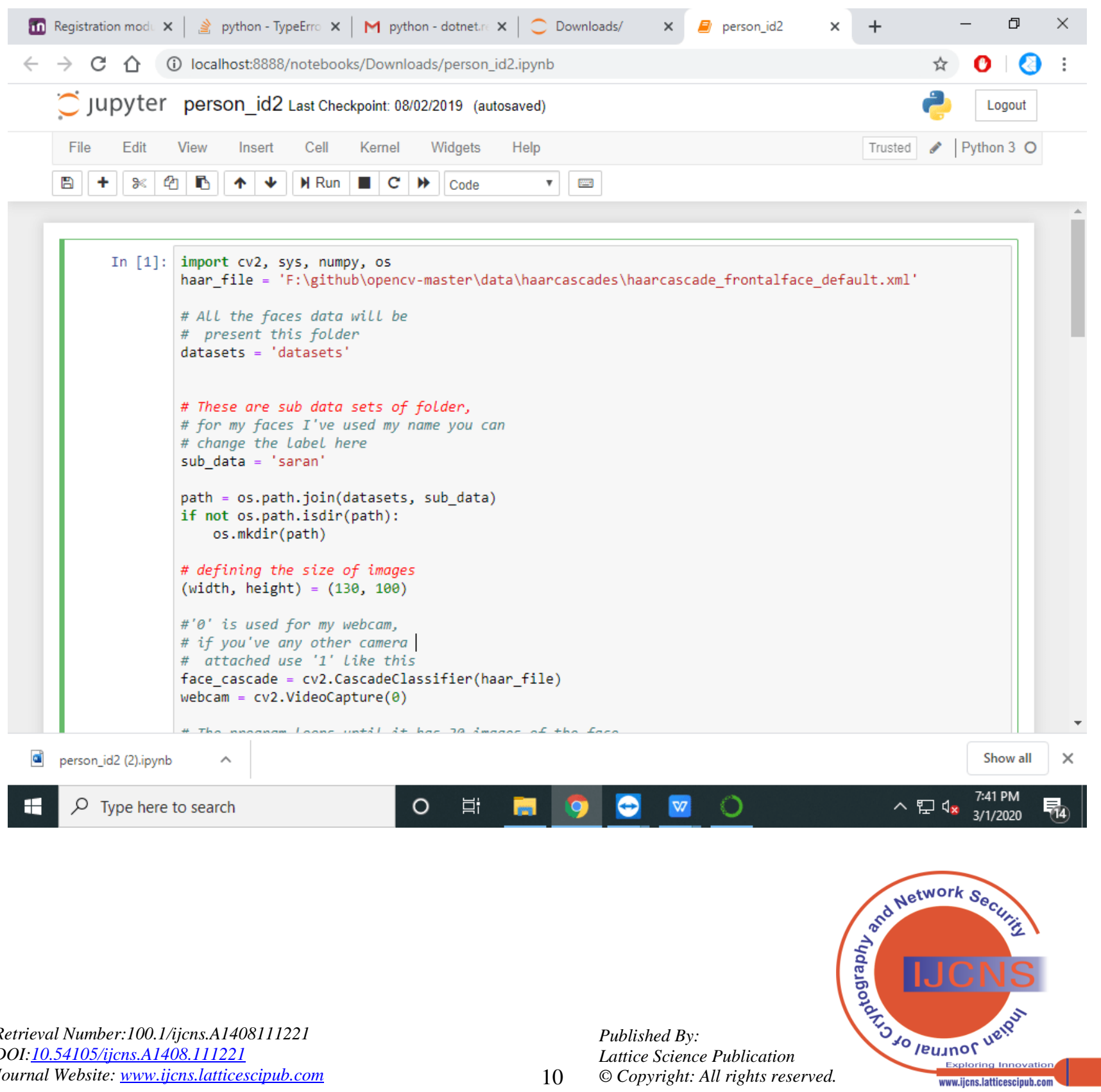


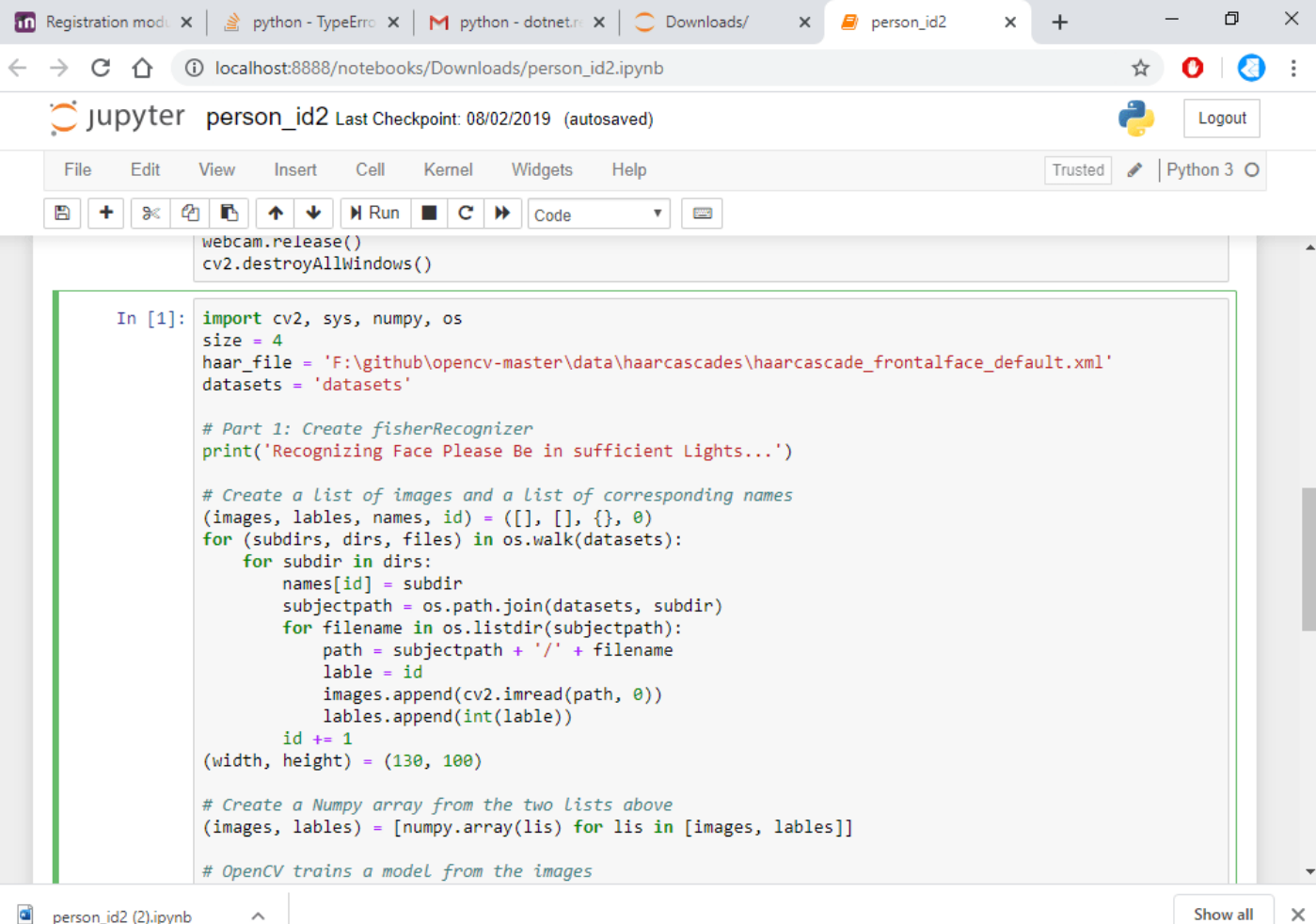

\# 0 Type here to search

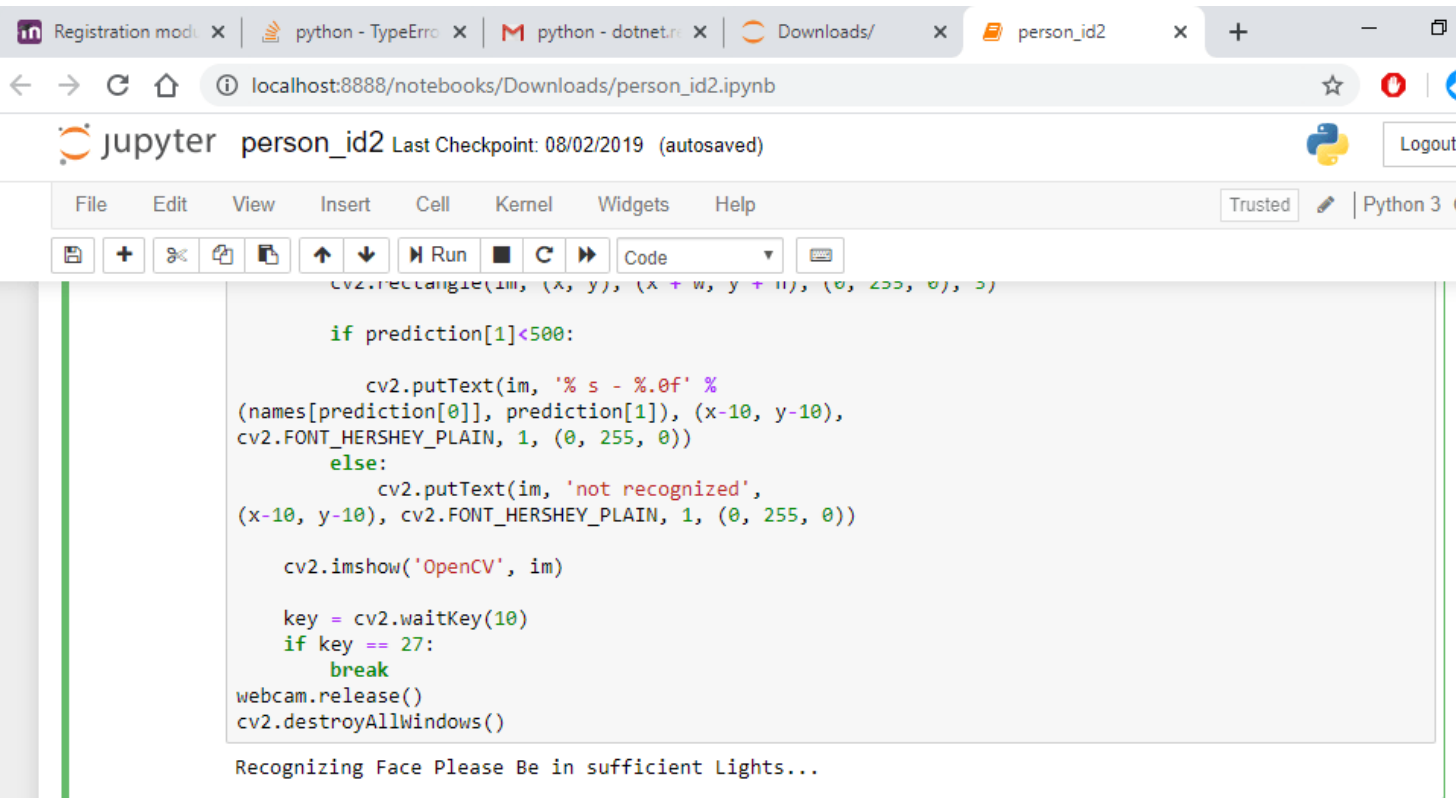

In [ ]:

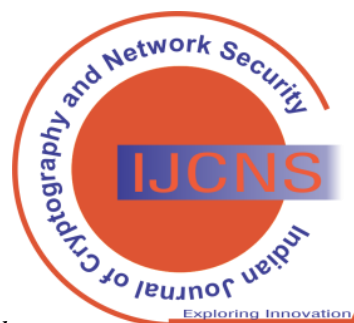




\section{Secure Online Transaction using Iris}

Enter your name: gopal

Enter password123

Enter your Email Address: saransamy64@ggmail.com

Enter your phone NNo: 9092898115

Set your pin...

Enter your PIN: 2902

Register successfully and here is your AccNO: 3049974284809242

\section{Login and transaction using pin}

Enter user name: ani

Enter password: 321

Enter account No: 5514028674478257

('ani', '321', 'dotnet.retech@gmail.com', '896574258', '5514028674478257', '1000', '4444')

would you like to transact the money: $y$

enter account no: 5514028674478257

enter account no: 640

enter receipient name: tharik

Enter amount: 200

would you like to proceed with authentication via (face or pin): pin

confirm your PIN: 4444

Transaction Done!

In 「 1:

Transaction using face Authentication

Enter user name: shahana

Enter password: 123

Enter account No: 1883979184945635

('shahana', '123', 'saransamy85@gmail.com', '9092098115', '1883979184945635', '1000', '2902')

would you like to transact the money: $y$

enter account no: 1883979184945635

enter account no: 3049974284009242

enter receipient name: gopal

Enter amount: 750

would you like to proceed with authentication via (face or pin): face

Recognizing Face Please Be in sufficient Lights...

transaction done!

your current Balance: ('1000', )

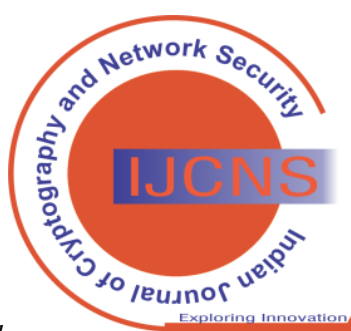




\section{TESTING SOFTWARE TESTING}

\section{A. General}

In a broad sense, we can define system testing as a type of testing whose primary goal is to ensure that a system runs smoothly and efficiently. The testing method is applied to a program with the goal of discovering an unheard-of fault, an error that could have harmed the software's future. Successful test cases are those that have a high chance of detecting and correcting errors. This successful test aids in the identification of errors that are still unknown.

\section{B. Test Case}

As previously said, testing is the process of identifying all probable flaws in the finalized software product. Testing aids in the verification of sub-assemblies, components, assembly, and the final product. The software executes a number of tests to guarantee that business needs and user expectations are not unexpectedly fulfilled. Today, a variety of tests are used. Each test type is designed to satisfy a distinct testing need.

\section{C. Testing Techniques}

A test plan is a document that outlines the method, scope, resources, and timeframe for conducting targeted testing sessions. It aids in the identification of nearly any other test item, the features to be tested, the activities to be performed, how everyone will perform each task, the tester's independence, and the setting in which the test is being conducted. Its design methodology, as well as the end criteria that are employed, as well as the rationale for their decision, and any type of danger that necessitates emergency preparedness. It can also be referred to as a record of the test planning process. In most cases, test plans are created with input from test engineers.

\section{A. UNIT TESTING}

Legal test planning includes unit testing to assist internal program logic verification. All of the decision branches as well as the internal code are validated. It occurs after each individual unit has been completed. It is also taken into consideration after the individual has been unified before integration. As a result, the unit test provides a basic level test at the component level, testing the specific business process, system configurations, and so on. It assures the precise defined specification of your own path of the testing unit with well documented inputs and anticipated outputs.

\section{E. B. INTEGRATION TESTING}

This checks are conducted to assess the real functionality or application of the application framework. Since evaluations are crucial to the activity, they focus on the fundamental conclusions of field work. Implementation tests indicate that the elements are pleased and are suitable and functioning, as demonstrated by successful testing process. This type of testing has been developed to uncover a combination of components that can lead to difficulties.

\section{F. C. FUNCTIONAL TESTING}

This testing can leverage the functionalities of the test and help to express precisely what functional specifications, design documents and design documentation describe.

\section{G. System Testing}

System testing, as its name implies, is the process of ensuring that a software system meets business goals and objectives. Here we test the configuration to provide predictable output and analyze the results. It focuses on the process integration point that is based on the system testing process and the definition of its flow and driven in advance.

\section{H. White Box Testing}

This Box Testing Analysis is a tool of analysis that testers are able to perform by disclosing the system's core components. As a result, it is a difficult testing procedure. To detect a possible problem or error, the tester tests the entire data structure, components, and so on. When the black box is unable to detect a bug, this method is employed. It is a more complicated sort of testing that takes longer to implement.

\section{Black Box Testing}

This Box analysis is a tool of application test that hides the critical processor architectures in order to find defects exclusively using system inputs and outputs. As a result, it is a straightforward type of testing. This form of testing can also be handled by a programmer with basic programming skills. When compared to white box testing, it takes less time. It works well for software that is less complicated and straightforward in nature. In addition, it is less expensive than white box testing.

\section{J. Acceptance Testing}

Tests for user approval is an essential feature of the project and include end users. The system also ensures that the operational requirements have been met.

\section{RESULTS AND DISCUSSION}

We used a facial recognition technology in this investigation to enable a secure and trustworthy bank transaction. The use of deep for facial authentication has proven to be successful in increasing the level of security when conducting banking transactions. With the employment networks for face authentication, it is envisaged that the security level of mobile banking would improve.

\section{CONCLUSION AND FUTURE WORK}

Machine learning was used to create a reliable, real-time facial recognition system. Various improvement has occurred in the new technological era, and some facial recognition algorithms have gained prominence. For face recognition, we use the Haar cascade technique. The capture module is in charge of video interface setting and real-time video capturing. Each captured frame is analyzed by the Face Detection module, which extracts valid faces from each frame. Face identification is the process of recognizing and verifying a face that has been detected. With the use of a radio frequency identification card, any fraudulent access by a false user will be eliminated in the future.

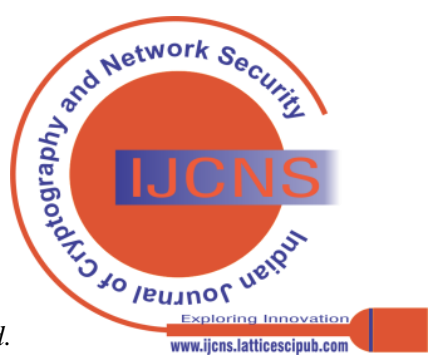




\section{Secure Online Transaction using Iris}

\section{REFERENCE}

1. Facial-Recognition Payment: An Example of Chinese Consumers, Wen Kun Zhang ; Min Jung Kang, IEEE Access, Year: 2019

2. Secure multifactor authentication payment system using NFC, Anirudhan Adukkathayar ; Gokul S Krishnan ; Rajashree Chinchole, 2015 10th International Conference on Computer Science \& Education (ICCSE)

3. Biometric Face Recognition Payment System, Surekha. R. Gondkar Saurab. Dr. C. S. Mala International Journal of Engineering Research \& Technology NCESC - 2018 Conference Proceedings

4. Facial Recognition in Banking - Current Applications, Niccolo Mejia,2019 Conference Proceedings

5. "Face Detection and Recognition for Bank Transaction ", International Journal of Emerging Technologies and Innovative Research, Sudarshan Dumbre, Shamita Kulkarni, Devashree Deshpande, P.V. Mulmule Journal of Emerging Technologies and Innovative Research 2018

6. Continuous User Identity Verification Using Biometric Traits for Secure Internet Services, Dr. SHAIK ADBUL MUZZER, 2GOSALA SUBHASIN

7. 7.Skin colour based Face detection Method, Devendra Singh Raghuvanshi, Dheeraj Agrawal

8. 8.Face Detection system based on retinal connected neural network (RCNN), Rowley, Baluja and Kanade 9.Combining Skin Colour based Classifiers and HAAR Feature using VJ Algorithm, N.Gobinathan, Abinaya and Geetha. P

9. 10.Face Detection and Recognition for Bank Transaction, Sudarshan Dumbre, Shamita Kulkarni, Devashree Deshpande, Prof P.V. Mulmule

\section{AUTHORS PROFILE}

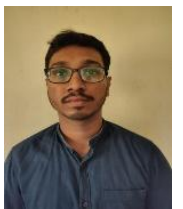

Myself Abhishek Balamurugan, B. Tech, Computer Science from SRM University Chennai I am a tech enthusiast who is keen to solve real world problems and that reflects through my projects like Smart Transportation system to the current research about "Secure Online Transaction Using IRIS “. I completed my primary education from ST Mary's High School, Tandur and my Intermediate Education at Bhashyam Junior College, Tandur through my projects and engineering journey I realized that I am quite good at ideation and implementation of various ideas and technologies and wish to solve more such public problems.

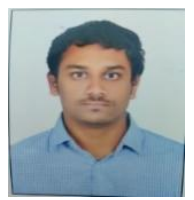

Myself Sai Dhanush. R, B. Tech, Computer Science from SRM University Chennai born and raised in Chennai, Tamilnadu. I completed my primary education from Jawahar Vidyalaya Senior Secondary School(CBSE), Ashok Nagar, Chennai and my Intermediate Education at Jawahar Higher Secondary School, Ashok Nagar, Chennai. through my projects and engineering journey I realized that I am quite good at ideation and implementation of various ideas Moving forward, I hope to expand my experience across different industries.

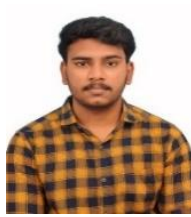

Myself Sundeep. J, B. Tech, Computer Science from SRM University Chennai born and raised in Chennai, Tamilnadu. I completed my primary education and Intermediate Education at Little Flower Matriculation Higher Secondary School, Ashok Nagar, Chennai. and it was the interest in programming and problem solving that brought me to the research field. Throughout this whole journey, I've noticed that doing what you are good at makes the best out of what you can do.

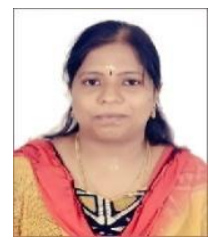

Myself Sivasankari. K, working as an Assistan professor in the department of CSE, SRM IST, Ramapuram Campus, Chennai. Completed my HSC in 2002 and my graduation in 2002-04 Anna University and then post graduated from Anna University in 2013. Having 10 years of teaching experience in various engineering colleges and have guided more than 20 batches in my career. I along with teaching subjects from curriculum also trey to inculcate research-based mindset in my students and promote problem solving approach in them that could lead them towards solving some real issues through their knowledge.

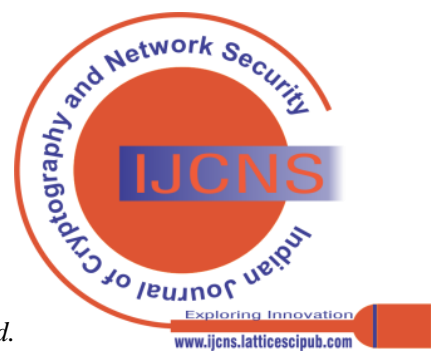

